\title{
A Study on the Benefit of Corporate Accounting Information in accordance with the Company's conservatism (from a management accounting consulting perspective)
}

Lee Soon Mi, Doctoral Student, Dept. Of Knowledge Service \& Consulting, Hansung University, Seoul, Korea, okdltnsal@naver.com

Yen-yooYou, Professor, Dept. Of Knowledge Service \& Consulting, Hansung University, Seoul, Korea, threey0818@hansung.ac.kr

*Corresponding Author

\begin{abstract}
This paper aims to judge the impact of accounting information on corporate profits based on conservatism. As a verification sample, 543 companies listed on securities (excluding KOSDAQ and financial industry) among the Bank of Korea (2018) "2017 Business Management Analysis" and the Korea Productivity Center's corporate analysis were sampled (December). Regression analysis was performed after analysis of search factors using SPSS22. Corporate accounting information. activity. Stable growth is positive as a financial analysis index that has a positive $(+)$ effect on profitability, corporate remuneration is profitable as an appropriate effect, and profitability is a positive (+) effect, profitability, activity and stability, prior research and research expenses. And savings will be reflected immediately. but will be affected in the worst case. The explanation of corporate accounting information is a financial performance that proves that corporate remuneration prepares for future uncertainty and provides profit benefits. In the future, it is necessary to discuss corporate compensation such as high value-added productivity as an additional variable of corporate accounting information.
\end{abstract}

Keywords: Corporate Accounting Information, Corporate conservatism, International Accounting St andards (IFRS), Profitability, Stability, Activity, Growth ability, Profitability Benefits Received: 08.12.2020 Accepted: 14.01 .2021 Published: 05.02.2021

\section{INTRODUCTION}

In recent prior research, the way companies evaluate companies and investors is mostly in two forms. One is a research on over-confidence bias[1] that is biased toward information asymmetry[2] and profit management, and the other is a study on transparency of accounting information [3] in dealing with costs and losses.

In this study, we analyzed whether the stronger the enterprise's conservatism, the more information asymmetry [4], is reflected in a way of profit growth that reflects the view that the better the quality of accounting information will be [8], and whether it is reflected in the manipulation of profits by institutional investors as part of accounting conservatism $[9,10]$. In addition, to reflect the characteristics of accounting remuneration and the Board of Directors, an entity's conservatism was used as a control variable to analyze its benefits $[13,14,15]$.

\section{Theory and formula}

\section{2-1-1 Corporate Accounting Information}

Unlike previous financial statements, an entity's accounting information assesses its profitability, stability, activity and growth in accounting. This assessment can also reduce the uncertainty of financial robustness and quality of accounting information coming from the asymmetry of information between special stakeholders and the benefit of business and investors in companies that introduce international accounting standards [2, 3]. 


\section{2-1-2 Corporate conservatism}

Corporate conservatism improves the quality of an entity's accounting information by preventing managers' opportunism and protecting special stakeholders and investors through the firm's financial robustness, evaluates losses and costs as quickly as possible, and slowly assesses profits and profits to reduce the entity's accounting information transparency and agent costs. In this study, prior research is used to analyze how enterprise conservatism is used as a control variable and is affected by benefit benefits $[1,4]$.

\section{2-1-3 Benefit Benefits}

The excessive retention of in-house reserves by many companies has recently been raised as a social problem. These problems are causing problems such as having to allocate appropriately to shareholders' demand returns as corporate profits increase, and the more conflicts between shareholders and creditors increase, the higher the tendency of corporate conservatism, causing other people's capital to fall. In this study, we would like to analyze why financial robustness is necessary for efficient benefit [15].

\section{2-2-1 Research Method}

In the enterprise's accounting information, profitability shall be the ratio of operating capital operating profit, capital operating profit ratio, equity operating profit ratio, sales operating profit ratio, and activity shall be the ratio of operating capital turnover, fixed asset turnover rate and total capital investment rate. Stability is fixed ratio, fixed long-term suitability rate, debt ratio, growth rate shall be total capital increase rate, tangible fixed asset growth rate and equity capital increase rate, and the company's conservatism, which is the adjustment variable, is labor cost ratio, sales cost and general management cost, value added rate. We would like to study how corporate accounting information affects benefit as a result of the remuneration of the entity, with the dependent variable benefit being dividend propensity, dividend rate and equity dividend rate.

\section{2-2-2 Measurement Method}

With corporate conservatism as an adjustment variable, how the accounting information of the entity affects its benefit is analyzed in spss22 from a management accounting consulting perspective through an exploratory factor analysis and regression analysis.

\section{Experimental setup}

\subsection{Research Model}

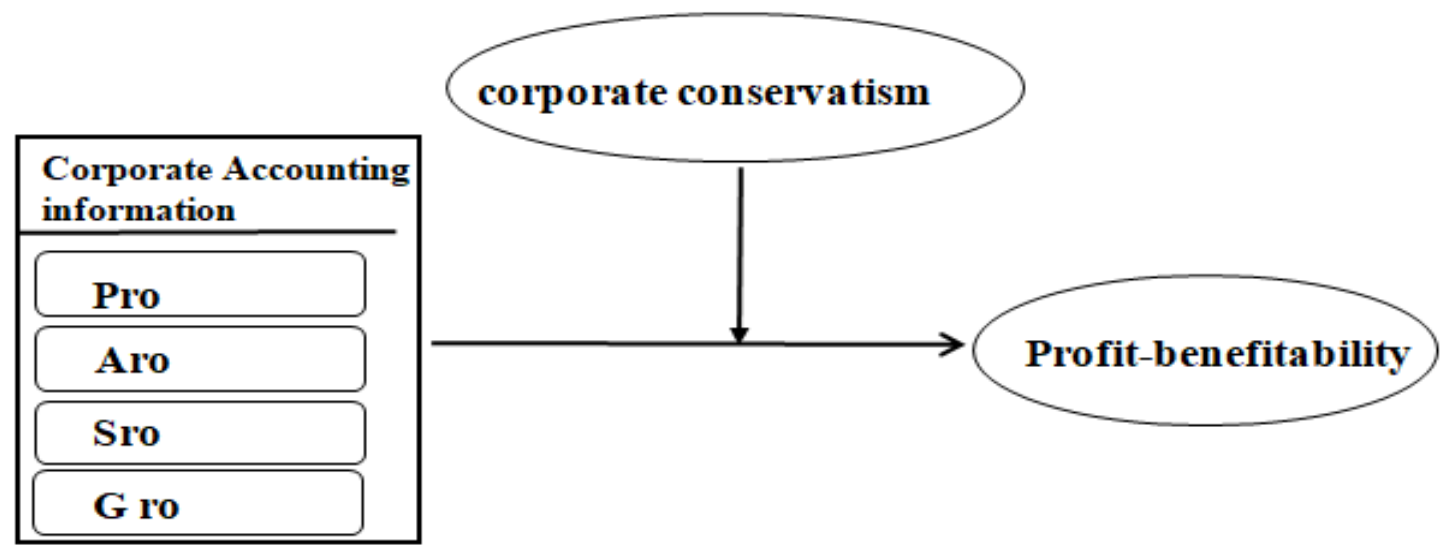

Figure1.ResearchModel

[Table 1] Descriptive Statistics

\begin{tabular}{|c|c|c|c|c|c|c|c|}
\hline N-o & $\begin{array}{l}\text { Minimu } \\
\text { m-0 }\end{array}$ & $\begin{array}{l}\text { Maximu } \\
\text { m-0 }\end{array}$ & Mean-0 & $\begin{array}{l}\text { Std } \\
\text { Deviatio } \\
\text { n-0 }\end{array}$ & Skewness-0 & Kurtosis & \\
\hline $\begin{array}{l}\text { Statisti } \\
\text { c }\end{array}$ & Statistic & Statistic & $\begin{array}{l}\text { Statist } \\
\text { ic }\end{array}$ & Statistic & $\begin{array}{ll}\text { Statisti } & \text { Std- } \\
\text { c } & \text { Error }\end{array}$ & $\begin{array}{l}\text { Statisti } \\
\text { c }\end{array}$ & $\begin{array}{l}\text { Sd- } \\
\text { Error }\end{array}$ \\
\hline
\end{tabular}




\begin{tabular}{|c|c|c|c|c|c|c|c|c|c|}
\hline Pro1 & 543 & -3.912 & 5.734 & $\begin{array}{l}1.2392 \\
3\end{array}$ & 1.193994 & -.314 & .105 & .674 & .209 \\
\hline Pro2 & 543 & -4.605 & 3.863 & 95969 & 1.086755 & -.617 & .105 & 1.377 & .209 \\
\hline Pro3 & 543 & -3.507 & 4.476 & $\begin{array}{l}1.4248 \\
2\end{array}$ & 1.217964 & -.393 & .105 & -.208 & .209 \\
\hline Pro4 & 543 & -3.912 & 4.401 & $\begin{array}{l}1.1398 \\
7\end{array}$ & 1.206010 & -.206 & .105 & .864 & .209 \\
\hline Aro1 & 543 & -4.605 & 1.267 & -.31792 & .781031 & -1.927 & .105 & 6.631 & .209 \\
\hline Aro2 & 543 & -3.507 & 1.890 & .03098 & .623925 & -.983 & .105 & 3.251 & .209 \\
\hline Aro3 & 543 & -4.605 & 3.547 & .28795 & .960450 & -1.278 & .105 & 4.636 & .209 \\
\hline Aro4 & 543 & -3.507 & 5.292 & $\begin{array}{l}2.5373 \\
3\end{array}$ & .867988 & -1.481 & .105 & 6.038 & .209 \\
\hline Sro1 & 543 & .445 & 7.088 & $\begin{array}{l}4.5778 \\
0\end{array}$ & .549605 & -.746 & .105 & 6.585 & .209 \\
\hline Sro2 & 542 & -4.605 & 2.570 & .09196 & 1.229287 & -1.934 & .105 & 4.490 & .209 \\
\hline Sro3 & 543 & -2.813 & 11.327 & $\begin{array}{l}4.0929 \\
6\end{array}$ & 1.105357 & -.436 & .105 & 6.350 & .209 \\
\hline Gro1 & 543 & -3.22 & 5.34 & .9832 & 1.28909 & .611 & .105 & .118 & .209 \\
\hline Gro2 & 542 & -3.22 & 7.37 & .8034 & 1.48636 & 1.211 & .105 & 1.745 & .209 \\
\hline Gro3 & 543 & -4.61 & 5.30 & 1.1024 & 1.36223 & .243 & .105 & .432 & .209 \\
\hline Cro1 & 543 & -.462 & 4.356 & $\begin{array}{l}2.3177 \\
0\end{array}$ & .738746 & -.530 & .105 & 1.361 & .209 \\
\hline Cro2 & 543 & -.073 & 4.770 & $\begin{array}{l}2.4195 \\
1\end{array}$ & .889732 & .158 & .105 & -.213 & .209 \\
\hline Cro3 & 543 & -1.897 & 4.580 & $\begin{array}{l}2.8409 \\
3\end{array}$ & .814926 & -1.326 & .105 & 4.354 & .209 \\
\hline Pbo1 & 543 & -1.05 & 7.92 & 2.0750 & 1.80101 & .082 & .105 & -1.169 & .209 \\
\hline Pbo2 & 543 & -3.51 & 10.67 & 2.1494 & 1.77956 & .302 & .105 & .066 & .209 \\
\hline Pbo3 & 543 & -4.61 & 3.10 & .2104 & .75836 & -.474 & .105 & 4.524 & .209 \\
\hline $\begin{array}{l}\text { Valid N } \\
\text { (listwise } \\
\text { ) } \\
\text { Results fre }\end{array}$ & 541 & & & & & & & & \\
\hline
\end{tabular}

[Table 2]Rotated Component Matrix a

\begin{tabular}{|c|c|c|c|c|c|c|c|}
\hline & \multicolumn{6}{|c|}{ Component } & \multirow[b]{2}{*}{ Cronbach } \\
\hline & $1-1$ & $2-1$ & $3-1$ & $4-1$ & $5-1$ & $6-1$ & \\
\hline Pro1 & .921 & & & & & & \\
\hline Pro2 & .909 & & & & & & \\
\hline Pro4 & .905 & & & & & & .967 \\
\hline Pro3 & .892 & & & & & & \\
\hline Aro1 & & .955 & & & & & \\
\hline Aro3 & & .913 & & & & & \\
\hline Aro2 & & .800 & & & & & .876 \\
\hline Aro4 & & .674 & & & & & \\
\hline Cro1 & & & .859 & & & & \\
\hline Cro3 & & & .770 & & & & .723 \\
\hline
\end{tabular}




\begin{tabular}{l|l|l|l|l|l|l|l} 
Cro2 & & & .619 & & & & \\
Pbo2 & & & & .818 & & & \\
Pbo1 & & & & .796 & & & \\
\hline Sbo3 & & & & .684 & & & \\
Sro3 & & & & & .882 & & .656 \\
Sro2 & & & & & .791 & & \\
\hline Gro1 & & & & & .683 & & \\
Gro2 & & & & & & .781 & \\
Gro3 & & & & & & .680 & \\
\hline Eigen-value & 4.028 & 3.414 & 2.253 & 2.216 & 2.062 & 1.733 & \\
\hline Variance(\%) & 20.14 & 17.071 & 11.266 & 10.63 & 10.31 & 8.666 & \\
\hline
\end{tabular}

KMO=.673 Bartlett's $=12238.626 . \mathbf{p . 0 0 0}$

Exploratory factor analysis for validity verification and berrymax rotation method analysis result for reliability verification $\mathrm{KMO}=.684$, Bartlett's $=12773.4 \mathrm{df}=190 \mathrm{p}>.000$, there was no problem in validity, and $79.84 \%$ of total explanatory power satisfies the conditions of multiple regression analysis [Table 2]

\subsection{Research theory}

Conservative accounting in enterprises has established $\mathrm{H} 1$ and research theory on the basis of preceding studies that are affected by financial information.

(H1) Effectiveness, stability, growth potential, and activity, which are measures of profitable accounting information, will have a positive (+) effect on corporate will.

Research hypothesis $\mathrm{H} 2$ was established with $\mathrm{H} 2$ based on previous studies in which corporate conservative accounting reflects profits and profits late.

(H2) Measured effect of profitable accounting information Phosphorus will give positive (+) profit to the profit of the company according to the conservatism of the company.

The research hypothesis $\mathrm{H} 3$ was established with $\mathrm{H} 3$ based on previous studies in which corporate conservatism responded more sensitively to cost rather than rotational power.

(H3) Activity, a measurement variable for accounting information, will affect the negative (-) of an entity's profit benefit in accordance with its conservatism.

Research hypothesis $\mathrm{H} 4$ was set up with $\mathrm{H} 4$ based on previous studies that corporate conservatism was immediately reflected only in costs and profits.

(H4) Stability, which is a measure of accounting information, will have a negative (-) effect on corporate profits and profits according to corporate conservatism.

The research hypothesis $\mathrm{H} 5$ was established as $\mathrm{H} 5$ based on previous studies that the conservatism of a company affects the capital structure of a company due to information asymmetry between stakeholders, which is a future growth power.

(H5) Growth, which is a measurement variable of accounting information, will have a negative (-) effect on corporate profits and profits according to corporate conservatism.

[Table 3]Model Summary ${ }^{b}$

\begin{tabular}{|c|c|c|c|c|c|c|c|c|c|c|}
\hline \multirow[b]{2}{*}{$\begin{array}{l}\text { Model- } \\
0 \\
\end{array}$} & \multirow[b]{2}{*}{$\mathrm{R}$} & \multirow[b]{2}{*}{$\begin{array}{l}\mathrm{R} \\
\text { Square } \\
\end{array}$} & \multirow[b]{2}{*}{$\begin{array}{l}\text { Adjusted } \\
\text { R Square }\end{array}$} & \multirow{2}{*}{$\begin{array}{l}\text { Std } \\
\text { Error of } \\
\text { the } \\
\text { Estimate- } \\
0\end{array}$} & \multicolumn{5}{|c|}{ Change Statistics- 0} & \multirow[b]{2}{*}{$\begin{array}{l}\text { Durbin } \\
\text { Watson- } \\
0\end{array}$} \\
\hline & & & & & $\begin{array}{l}\mathrm{R} \\
\text { Square } \\
\text { Change }\end{array}$ & $\begin{array}{l}\mathrm{F} \\
\text { Change }\end{array}$ & df1-0 & df2-0 & $\begin{array}{l}\text { Sig F } \\
\text { Change }\end{array}$ & \\
\hline 1 & $.533^{\mathrm{a}}$ & .284 & .279 & .69981 & .284 & 53.233 & 4 & 536 & .000 & 1.955 \\
\hline
\end{tabular}


[Table 4]Coefficients ${ }^{\text {a }}$

\begin{tabular}{|c|c|c|c|c|c|c|c|c|c|}
\hline \multirow[b]{2}{*}{ Model-0 } & \multicolumn{2}{|c|}{$\begin{array}{l}\text { Unstandardized } \\
\text { Coefficients- } 0\end{array}$} & \multirow{2}{*}{$\begin{array}{l}\begin{array}{l}\text { Standardized } \\
\text { Coefficients-0 }\end{array} \\
\text { Beta }\end{array}$} & \multirow[b]{2}{*}{$\mathrm{t}$} & \multirow[b]{2}{*}{ Sig. } & \multicolumn{2}{|c|}{$\begin{array}{l}\text { (95\%) Confidence } \\
\text { Interval for B-0 }\end{array}$} & \multicolumn{2}{|l|}{$\begin{array}{l}\text { Collinearity } \\
\text { Statistics-0 }\end{array}$} \\
\hline & B & $\begin{array}{l}\text { Std. } \\
\text { Error }\end{array}$ & & & & $\begin{array}{l}\text { Lower } \\
\text { Bound- } \\
0\end{array}$ & \begin{tabular}{|l} 
Upper \\
Bound-0 \\
\end{tabular} & $\begin{array}{l}\text { Tolerance- } \\
0\end{array}$ & VIF-0 \\
\hline 1 (Constant) & .000 & .030 & & -.010 & .992 & -.059 & .059 & & \\
\hline Pro & .394 & .035 & .457 & 11.251 & .000 & .325 & .463 & .809 & 1.236 \\
\hline Aro & .075 & .037 & .077 & 2.010 & .045 & .002 & .147 & .920 & 1.087 \\
\hline Sro & -.242 & .038 & -.237 & -6.415 & .000 & -.316 & -.168 & .981 & 1.019 \\
\hline Gro & -.108 & .043 & -.098 & -2.497 & .013 & -.193 & -.023 & .866 & 1.154 \\
\hline
\end{tabular}

Looking at the results of Table 3, Table 4 found that there was no problem with multicollinearity, profitability ( $\mathrm{t}$ 11.251.P .000), stability ( $\mathrm{t}$-6.415.p. .000), and growth potential ( $\mathrm{t}-2.497 . \mathrm{P} .013$ ) and activity $(\mathrm{t}-2.010$. $\mathrm{P}$.045) were also statistically significant, and the study hypothesis $\mathrm{H} 1$ was accepted.

[Table 5]Model Summary ${ }^{d}$

\begin{tabular}{|c|c|c|c|c|c|c|c|c|c|c|}
\hline \multirow[b]{2}{*}{$\begin{array}{l}\text { Model- } \\
0\end{array}$} & \multirow[b]{2}{*}{$\mathrm{R}$} & \multirow[b]{2}{*}{ RSquare } & \multirow[b]{2}{*}{$\begin{array}{l}\text { Adjusted } \\
\mathrm{R} \\
\text { Square }\end{array}$} & \multirow[b]{2}{*}{$\begin{array}{l}\text { Std Error of } \\
\text { the Estimate- } \\
0\end{array}$} & \multicolumn{5}{|c|}{ Change Statistics-0 } & \multirow[b]{2}{*}{$\begin{array}{l}\text { Durbin } \\
\text { Watson- } \\
0\end{array}$} \\
\hline & & & & & $\begin{array}{l}\mathrm{K} \\
\text { Square } \\
\text { Change }\end{array}$ & $\begin{array}{l}\text { F } \\
\text { Change }\end{array}$ & df1-0 & df2-0 & $\begin{array}{l}\text { Sig- F } \\
\text { Change }\end{array}$ & \\
\hline 1 & $.468^{\mathrm{a}}$ & .219 & .218 & .72922 & .219 & 151.817 & 1 & 541 & .000 & \\
\hline 2 & $.479^{b}$ & .229 & .227 & .72507 & .010 & 7.223 & 1 & 540 & .007 & \\
\hline 3 & $.498^{c}$ & .248 & .243 & .71711 & .018 & 13.054 & 1 & 539 & .000 & 1.984 \\
\hline
\end{tabular}

[Table 6]Coefficients a

\begin{tabular}{|c|c|c|c|c|c|c|c|c|}
\hline \multirow{2}{*}{\multicolumn{2}{|c|}{ Mode- 0}} & \multicolumn{2}{|c|}{$\begin{array}{l}\text { Unstandardized } \\
\text { Coefficients-0 }\end{array}$} & \multirow{2}{*}{$\begin{array}{l}\text { Standardized } \\
\text { Coefficients- } 0\end{array}$} & \multirow[t]{2}{*}{$\mathrm{t}$} & \multirow{2}{*}{ Sig. } & \multicolumn{2}{|l|}{$\begin{array}{l}\text { Collinearity } \\
\text { Statistics- } 0\end{array}$} \\
\hline & & $\mathrm{B}$ & $\begin{array}{l}\text { Std. } \\
\text { Error }\end{array}$ & & & & $\begin{array}{l}\text { Tolerance- } \\
0\end{array}$ & VIF-0 \\
\hline \multirow[t]{2}{*}{1} & (Constant) & $\begin{array}{l}4.312 \mathrm{E}- \\
16\end{array}$ & .031 & & & & & \\
\hline & Pro & .404 & .033 & .468 & 12.321 & .000 & 1.000 & 1.000 \\
\hline \multirow[t]{3}{*}{2} & (Constant) & $\begin{array}{l}3.639 \mathrm{E}- \\
16\end{array}$ & .031 & & .000 & 1.000 & & \\
\hline & Pro & .391 & .033 & .454 & 11.889 & .000 & .980 & 1.020 \\
\hline & Cro & .105 & .039 & .103 & 2.688 & .007 & .980 & 1.020 \\
\hline \multirow[t]{4}{*}{3} & (Constant) & -.017 & .031 & & -.533 & .594 & & \\
\hline & Pro & .379 & .033 & .440 & 11.603 & .000 & .970 & 1.030 \\
\hline & Cro & .114 & .039 & .112 & 2.959 & .003 & .975 & 1.025 \\
\hline & MPro & .153 & .042 & .136 & 3.613 & .000 & .987 & 1.013 \\
\hline
\end{tabular}

Looking at the results of Table 5. Table 6 found that the research hypothesis H2 that profitability among the measuring variables of accounting information is recognized lately in reflecting financial accounting due to corporate conservatism, which is a controlling variable. 
[Table 7]Coefficients a

\begin{tabular}{|c|c|c|c|c|c|c|c|}
\hline \multirow[b]{2}{*}{ Model-0 } & \multicolumn{2}{|c|}{$\begin{array}{l}\text { Unstandardized } \\
\text { Coefficients- } 0\end{array}$} & \multirow{2}{*}{$\begin{array}{l}\begin{array}{l}\text { Standardized } \\
\text { Coefficients-0 }\end{array} \\
\text { Beta-0 } \\
\end{array}$} & \multirow{2}{*}{$\mathrm{t}$} & \multirow{2}{*}{ Sig. } & \multicolumn{2}{|l|}{$\begin{array}{l}\text { Collinearity } \\
\text { Statistics-0 }\end{array}$} \\
\hline & $\mathrm{B}$ & $\begin{array}{l}\text { Std. } \\
\text { Error }\end{array}$ & & & & $\begin{array}{l}\text { Tolerance- } \\
0\end{array}$ & VIF-0 \\
\hline \multirow[t]{2}{*}{1 (Constant) } & $\begin{array}{l}4.782 \mathrm{E}- \\
16\end{array}$ & .035 & & .000 & 1.000 & & \\
\hline & .170 & .040 & .179 & 4.221 & .000 & 1.000 & 1.000 \\
\hline \multirow[t]{3}{*}{$\begin{array}{ll}2 & \text { (Constant) }\end{array}$} & $\begin{array}{l}3.425 \mathrm{E}- \\
16\end{array}$ & .034 & & .000 & 1.000 & & \\
\hline & .239 & .041 & .251 & 5.811 & .000 & .911 & 1.098 \\
\hline & .247 & .044 & .241 & 5.597 & .000 & .911 & 1.098 \\
\hline \multirow{4}{*}{ MAro } & -.009 & .035 & & -.260 & .795 & & \\
\hline & .260 & .045 & .273 & 5.827 & .000 & .774 & 1.292 \\
\hline & .253 & .044 & .248 & 5.708 & .000 & .896 & 1.116 \\
\hline & -.043 & .036 & -.054 & -1.207 & .228 & .850 & 1.177 \\
\hline
\end{tabular}

Looking at the results of Table 7 found that among the measuring variables of accounting information, the research hypothesis $\mathrm{H} 3$ was accepted that the conservatism of firms, which is a controlling variable, was not affected by profit-benefit.

[Table 8]Coefficients ${ }^{\text {a }}$

\begin{tabular}{|c|c|c|c|c|c|c|c|c|}
\hline \multirow{2}{*}{\multicolumn{2}{|c|}{ Model-0 }} & \multicolumn{2}{|c|}{$\begin{array}{l}\text { Unstandardized } \\
\text { Coefficients-0 }\end{array}$} & \multirow{2}{*}{$\begin{array}{l}\text { Standardized } \\
\text { Coefficients-0 }\end{array}$} & \multirow[t]{2}{*}{$\mathrm{t}$} & \multirow[t]{2}{*}{ Sig. } & \multicolumn{2}{|l|}{$\begin{array}{l}\text { Collinearity } \\
\text { Statistic-0 }\end{array}$} \\
\hline & & B & Std. Error & & & & $\begin{array}{l}\text { Tolerance- } \\
0\end{array}$ & VIF-0 \\
\hline \multirow[t]{2}{*}{1} & (Constant) & -.001 & .034 & & -.038 & .969 & & \\
\hline & Sro & -.284 & .042 & -.278 & -6.716 & .000 & 1.000 & 1.000 \\
\hline \multirow[t]{3}{*}{2} & (Constant) & -.001 & .034 & & -.041 & .967 & & \\
\hline & Sro & -.261 & .043 & -.255 & -6.074 & .000 & .961 & 1.040 \\
\hline & Cro & .120 & .043 & .117 & 2.799 & .005 & .961 & 1.040 \\
\hline \multirow[t]{4}{*}{3} & (Constant) & .001 & .034 & & .026 & .980 & & \\
\hline & Sro & -.261 & .043 & -.254 & -6.062 & .000 & .961 & 1.041 \\
\hline & Cro & .119 & .043 & .117 & 2.782 & .006 & .960 & 1.042 \\
\hline & MSro & .018 & .050 & .015 & .355 & .723 & .998 & 1.002 \\
\hline
\end{tabular}

Looking at the results of Table 8, it was found that among the measuring variables of accounting information, research hypothesis $\mathrm{H} 4$ was accepted that the stability of the controlling variable, corporate conservatism, was not affected by the profit-benefit.

[Table 9]Coefficients a

\begin{tabular}{|c|c|c|c|c|c|c|c|}
\hline \multirow[b]{2}{*}{ Model-0 } & \multicolumn{2}{|c|}{$\begin{array}{l}\text { Unstandardized } \\
\text { Coefficients-0 }\end{array}$} & \multirow{2}{*}{ 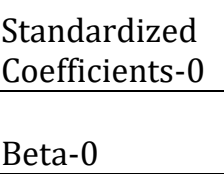 } & \multirow{2}{*}{$\mathrm{t}$} & \multirow{2}{*}{ Sig. } & \multicolumn{2}{|l|}{$\begin{array}{l}\text { Collinearity } \\
\text { Statistics-0 }\end{array}$} \\
\hline & B & Std. Error & & & & $\begin{array}{l}\text { Tolerance- } \\
0\end{array}$ & VIF-0 \\
\hline 1 (Constant) & .002 & .035 & & .043 & .965 & & \\
\hline Gro & .088 & .047 & .080 & 1.872 & .062 & 1.000 & 1.000 \\
\hline
\end{tabular}




\begin{tabular}{ll|l|l|l|l|l|l|l}
2 & (Constant) & .002 & .035 & & .062 & .950 & & \\
& Gro & .087 & .047 & .079 & 1.872 & .062 & 1.000 & 1.000 \\
& Cro & .176 & .043 & .172 & 4.067 & .000 & 1.000 & 1.000 \\
\hline 3 & (Constant) & .002 & .035 & & .062 & .951 & & \\
& .087 & .047 & .079 & 1.857 & .064 & .991 & 1.009 \\
& Gro & .176 & .043 & .172 & 4.063 & .000 & 1.000 & 1.000 \\
Cro & .003 & .059 & .002 & .056 & .956 & .991 & 1.009 \\
\hline
\end{tabular}

Looking at the results of Table 9 found that among the measuring variables of accounting information, research hypothesis $\mathrm{H} 5$ was accepted that firm's conservatism, which is a controlling variable for growth, was not affected by profit-benefit.

[Table 10]Coefficients a

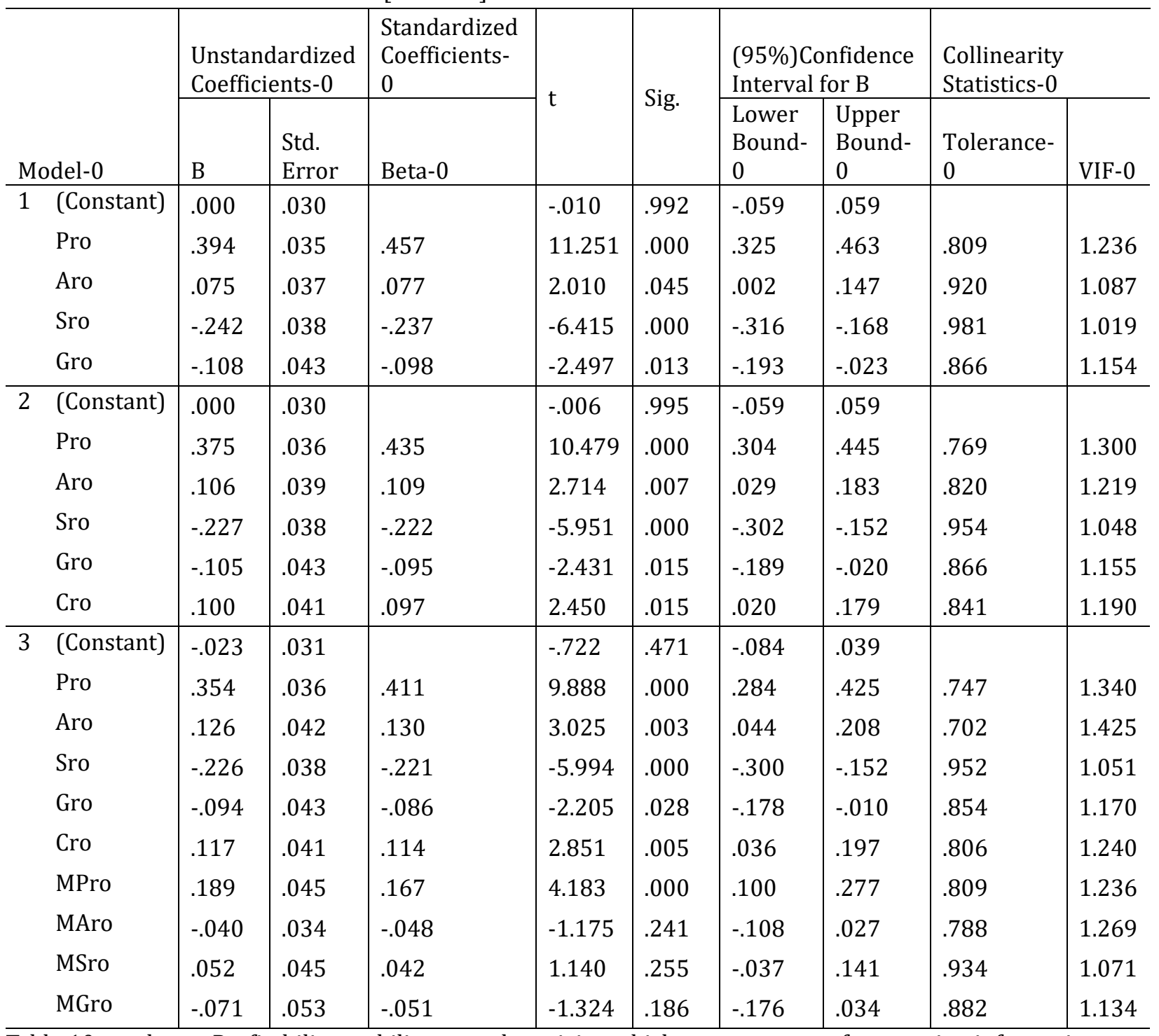

Table 10 results areProfitability, stability, growth, activity, which are measures of accounting information, were conducted to verify the adjustment variables in the effects of the entity's profit-benefit. Step 1 validated the effect on profit benefit as an independent variable, which is a measure of accounting information, step 2 used the entity's conservatism, which is an adjustment variable, and step 3 used the interaction variable between the independent variable and the modifier. Therefore, it was found that corporate remuneration reliably affects the financial structure of a company for costs and losses rather 
than overconfidence of managers, and it affects the economic value of a company by reflecting the profitability and profits in the financial accounting late. It also showed that activity, stability, growth do not have a significant impact on corporate remuneration due to future growth, capital structure, and corporate financial turnover.

\section{Result discussions}

[Table11]Result of research

\begin{tabular}{|c|c|c|}
\hline & Hypothesis & Result \\
\hline $\mathbf{H}$ & $\begin{array}{l}\text { A Study on the Benefit of Corporate Accounting Information } \\
\text { accordance with Corporate Conservatism }\end{array}$ & \\
\hline $\mathrm{H} 1$ & $\begin{array}{l}\text { The entity`s accounting information is affected by the by the amount } \\
\text { of profit-benefit }(+)\end{array}$ & Accept \\
\hline $\mathrm{H} 2$ & $\begin{array}{l}\text { Profitability is corporate conservatism }(+) \text { Therefore, I get positive } \\
(+) \text { for profit-benefit }\end{array}$ & Accept \\
\hline $\mathrm{H} 3$ & $\begin{array}{l}\text { Activity is corporate conservatism Therefore, it receives negative }(-) \\
\text { in profit-benefit }\end{array}$ & Accept \\
\hline $\mathrm{H} 4$ & $\begin{array}{l}\text { Stability is corporate conservatism Therefore, it receives negative }(-) \\
\text { in profit-benefit }\end{array}$ & Accept \\
\hline H5 & $\begin{array}{l}\text { Growth is a corporate conservatism. Therefore, it receives negative } \\
(-) \text { in profit-benefit }\end{array}$ & Accept \\
\hline
\end{tabular}

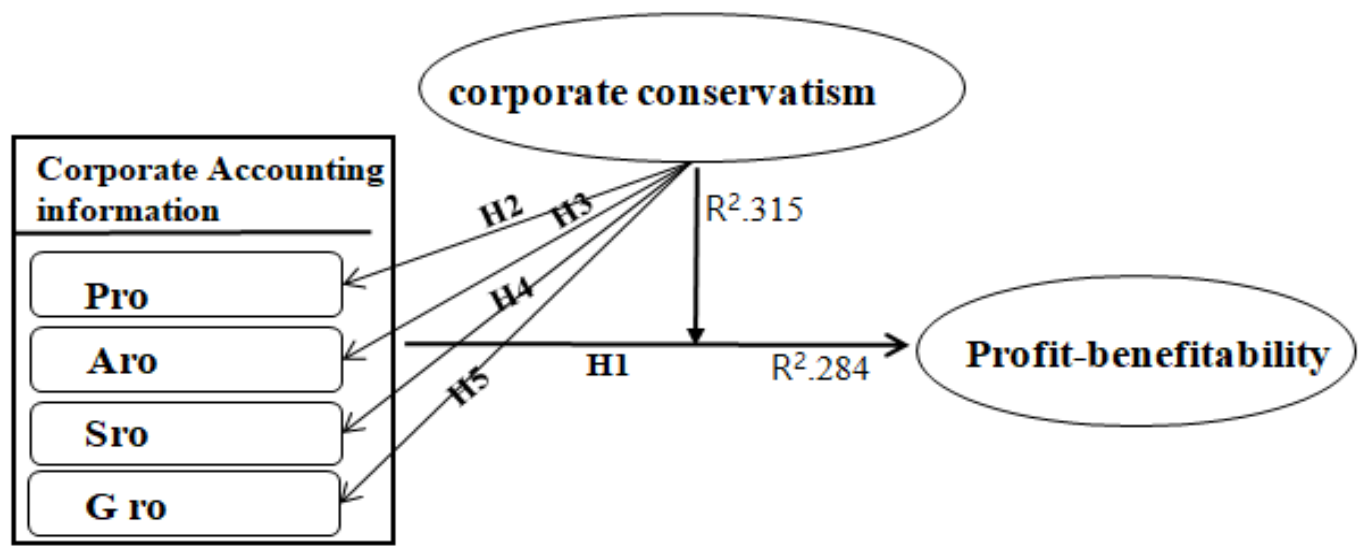

Figure2. Research Model

\section{Conclusions}

The measured variables such as profitability, activity, stability, and growth were found to have a positive $(+)$ effect on profit benefit. In addition, it was found that corporate conservatism had a positive (+) effect on profitability. the research hypothesis that costs and losses are immediately reflected, but profits and profits are reflected late in preparation for future uncertainty, was proven by the measurement of corporate conservative accounting information. In addition, the results of the profit benefit analysis showed that the appropriate line of dividends between the company's excessive in-house reserves and shareholders and investors was proven as a measurement variable. In this study, we also looked at how agency costs between enterprises and managers and information asymmetry between enterprises and investors affect benefits from corporate conservatism. In the future, discussions are needed to compare the dividend policy profit benefit to corporate conservatism by adding corporate accounting information 
and high value-added productivity

\section{Acknowledgements}

This research was financially supported by Han sung University.

\section{References}

[1] Gor, Yusuf, Rifat Karakus, and Izzet Tasar. "Conservatism, corporate governance and audit quality: A study at Istanbul Stock Exchange." Theoretical \& Applied Economics $24 \quad$ (2017).

[2] Ryu, Haeyoung. "The Effect of Managerial Overconfidence on Crash Risk." The Journal of Industrial Distribution \& Business 8, no. 5 (2017): 87-93.

[3] Bushman, Robert M., and Abbie J. Smith. "Transparency, financial accounting information, and corporate governance." Financial accounting information, and corporate governance. Economic Policy Review 9, no. 1 (2003).

[4] Basu, Sudipta. "The conservatism principle and the asymmetric timeliness of earnings1." Journal of accounting and economics 24, no. 1 (1997): 3-37.

[5] Chi, Sung-Kwon. "External financing constraints and Dividend Policy in accordance with the ownership structure." Management \& Information Systems Review 33, no. 5 (2014): 171-184.

[6] LaFond, Ryan. "The influence of ownership structure on earnings conservatism and the informativeness of stock prices: An international comparison." Sloan School of Management Working Paper (2005).

[7] Byun, Sun-Young, Jin-Wook Kim, and Hyun-Uk Jung. "Tax Subsidy and Information Effect of Future Earnings." Management \& Information Systems Review 35, no. 4 (2016): 123-140.

[8] Collins, Daniel W., and Linda DeAngelo. "Accounting information and corporate governance: Market and analyst reactions to earnings of firms engaged in proxy contests." Journal of Accounting and Economics 13, no. 3 (1990): 213-247.

[9] Lin, Fengyi, Chung-Min Wu, Tzu-Yi Fang, and Jheng-Ci Wun. "The relations among accounting conservatism, institutional investors and earnings manipulation." Economic Modelling 37 (2014): 164174.

[10] Lin, Ling, and Pavinee Manowan. "Institutional ownership composition and earnings management." Review of Pacific Basin Financial Markets and Policies 15, no. 04 (2012): 1250022.

[11] Gordon, Lawrence A., and Danny Miller. "A contingency framework for the design of accounting information systems." In Readings in accounting for management control, pp. 569-585. Springer, Boston, MA, 1976.

[12] Mora, Araceli, and Martin Walker. "The implications of research on accounting conservatism for accounting standard setting." Accounting and Business Research 45, no. 5 (2015): 620-650.

[13] Park, Chan-Jung, and Kyu-Chan Lim. "The Relationship Between Information Technology Structure and Management Accounting Information in AIS." The Journal of the Korea Contents Association 6, no. 7 (2006): 31-41.

[14] Kim, Dong-Il. "Analysis on Chinese companies with Introduction of the IFRS and the Conservatism Features." Journal of Digital Convergence 14, no. 8 (2016): 105-113.

[15] Kong, Xiangting, Suresh Radhakrishnan, and Albert Tsang. "Corporate lobbying, visibility and accounting conservatism." Journal of Business Finance \& Accounting 44, no. 5-6 (2017): 527- 